\title{
VARIABILITY OF THE OORT CLOUD COMET FLUX: CAN IT BE MANIFEST IN THE CRATERING RECORD?
}

\author{
J.J. MATESE AND P.G. WHITMAN \\ Department of Physics, The University of Southwestern Louisiana \\ Lafayette, Louisiana, 70504-4210 USA \\ K.A. INNANEN \\ Department of Physics and Astronomy, York University, Ontario, Canada \\ AND \\ M.J. VALTONEN \\ Tuorla Observatory, Turku University, Finland
}

\begin{abstract}
We consider the subject of time dependence of the Oort cloud comet flux. Over long time scales the flux is likely to be dominated by the adiabatic galactic tide. This tide is substantially modulated as the Solar System moves in its galactic orbit. If Shoemaker was correct in his estimate that virtually all terrestrial craters of diameter $>100 \mathrm{~km}$ are produced by long period comets, then the phase and plane crossing period of the Solar System about the galactic disk should be consistent with the ages of accurately dated large craters. A time series analysis of these ages in which the Solar oscillation phase is fixed to be consistent with observations indicates a maximal correlation for a period of $36 \pm 2 \mathrm{Myr}$. This period is well within observational limits. If improvements in stellar velocity dispersion studies continue, it is possible that a sufficiently accurate determination of the Solar oscillation period can be found to unambiguously answer the following questions. Is the Solar oscillation cycle correlated with the time series of ages for large craters? If so, can we reject the hypothesis that the correlation is an artifact that could likely be reproduced by a random distribution of ages? We present evidence which suggests that if it is found that the data requires a plane crossing period in the range $36 \pm 2 \mathrm{Myr}$, the answer to both of these questions will be affirmative.
\end{abstract}

\section{Introduction}

In the Rampino model (Rampino and Stothers, 1986; Rampino and Haggerty, 1996; Rampino et al., 1997), as the Sun oscillates about the galactic disk, the Oort cloud of comets is more likely to receive an impulse from a molecular cloud as it passed the galactic midplane than when it is at maximum displacement from the midplane. Thus we can expect a quasi-periodicity in the modulation of the probability of these events. It has been argued that these events are too rare and the probability modulation too broad (Thaddeus, 1986) to produce a detectable signal. Subsequently it was shown (Matese et al., 1995) that the continuous adiabatic tidal pertubation of the galaxy, which appears to dominate in making Oort cloud comets observable (Heisler, 1990), will undergo a similar modulation. But the objection that the modulation might be too broad remained.

Statistical arguments (Heisler and Tremaine, 1989; Grieve and Pesonen, 1996) indicated that for a small number of events (craters or extinctions), one could not expect a significant deduction about the reality of any putative period and phase if the convolution of dating uncertainties, period jitter and signal width was too large.

An entirely different problem consists of considering a mechanism (the production of long period comet impactors) that is known to be capable of producing these events and that is necessarily modulated quasi-periodically. The relevant question then is whether a known period and phase. correlates with the time series of the events in a significant manner. The importance of answering 
this question is that it will shed light on the dynamical mechanism which dominates in producing these events. It must be emphasized that this problem has not been addressed statistically because the period has not been, as yet, adequately determined.

We now anticipate this problem and provide an analysis that is meant to condition the debate which will ensue when the galactic oscillation period is accurately bounded.

\section{Analysis}

In Matese et al. (1995), it was demonstrated that a substantial modulation in the adiabatically induced Oort cloud comet flux is necessary if there is to be consistency with galactic disk mass limits implied by observations of stellar velocity dispersions. If no dark disk matter exists the plane crossing period will be $\approx 45 \mathrm{Myr}$ and the peak to trough flux variation will be $\approx 2.5$ to 1 . With a self consistent amount of compact dark disk mass, the period could be as low as $30 \mathrm{Myr}$ with a variation in flux of 4 to 1 . However periods that low or lower will imply that recent dark disk matter mass determinations are discrepant by more that $1 \sigma$ (Flynn and Fuchs, 1994). For discussion sake we shall allow that ultimately the period will be found to be somewhere in the range 25 Myr $\leq P \leq$ 45 Myr.

In determining a best correlated period we consider the set of craters satisfying the criteria that they are large (so that they will be dominated by long period comet impactors if Shoemaker et al. (1990) are correct), young (ages $\leq 250 \mathrm{Myr}$ so that erosion is minimized) and accurately dated (uncertainties $\leq 3 \mathrm{Myr}$, so that the statistical dispersion is dominated by the width of the cycle flux). We are left with the following 9 craters.

TABLE 1. Large Accurately Dated Craters

\begin{tabular}{|llcl|}
\hline Crater & $D(\mathrm{~km})$ & $T(\mathrm{Myr})$ & $\Delta T(\mathrm{Myr})$ \\
\hline Chicxulub & 180 & 64.98 & 0.05 \\
Morokweng & 100 & 145 & 3 \\
Manicouagan & 100 & 214 & 1 \\
Popagai & 100 & 35.7 & 0.8 \\
Chesapeake & 90 & 35.2 & 0.3 \\
Kara & 65 & 73 & 3 \\
Montagnais & 45 & 50.5 & 0.8 \\
Mjolnir & 40 & 142 & 2.6 \\
Manson & 35 & 73.8 & 0.3 \\
\hline
\end{tabular}

The analysis described makes no predictive claims as to the statistical significance of the best fit period obtained using this small number of craters. It will depend on the biases inherent in the interval choices for time, crater diameter and dating uncertainty. It will also depend on the cycle width of the putative dynamical mechanism. This does not mean that the results are without information content as we shall argue.

As a measure of goodness of fit we first determine the modulated flux produced by the galactic tide in a series of calculations in which the disk density is varied in such a way as to produce a sequence of flux versus time plots. The sequence gives the time dependent flux for 21 separate cases differing in mean plane crossing period by $1 \mathrm{Myr}$ in a range $25-45 \mathrm{Myr}$.

In Figure 1 we show the flux for a single case with a mean period of 36 Myr. The peak flux times lag the galactic plane crossing times by $\approx 2 \mathrm{Myr}$ and are not precisely periodic because of decreasing galactic density as the Sun recedes from the galactic core. The phase of the oscillations is restricted by observations which place the last previous plane crossing at $\approx 1$ Myr in the past (and the next flux peak $\approx 1 \mathrm{Myr}$ in the future). A perigalactic period of $\approx 180 \mathrm{Myr}$ is observable in the data. The background shown is a combination of the adiabatic tidal effects of the large scale height old star population as well as an assumed contribution from stellar impulses of the outer Oort cloud affecting comets not accessible to the galactic tide. We do not show the larger but rarer contributions due to random stellar-induced showers from the inner Oort cloud which are estimated to add $\approx 20 \%$ to the total but dominate only $2 \%$ of the time (Heisler, 1990). 


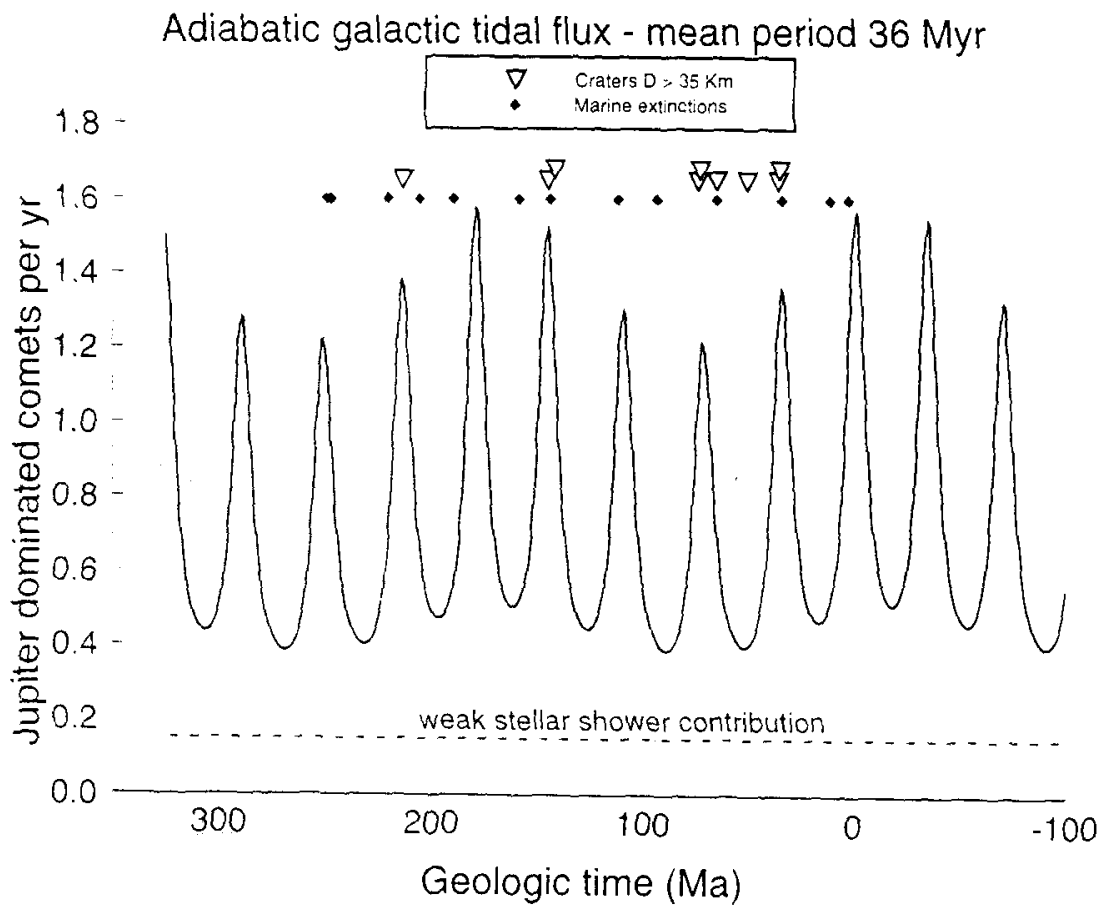

Figure 1. A model of the variable Oort cloud comet flux as modulated by the adiabatic galactic tide. Past time is positive here. Markers for 9 accurately dated large craters and 13 marine extinctions $\geq 20 \%$ are shown.

The peaks above the background are attributable to the adiabatic tide of the compact component of the disk composed of molecular clouds, dust and young stars. Random molecular cloud impulses of the Oort cloud will have their probabilities modulated is a manner similar to that shown. The standard deviations of the peaks are $\approx 4-5 \mathrm{Myr}$, but if the background is included the formal standard deviation of a complete cycle is closer to $7-8 \mathrm{Myr}$. That is, roughly $2 / 3$ of the model's flux occurs in a time interval of $\approx 15 \mathrm{Myr}$. Random strong stellar showers will broaden this interval while modulated strong molecular cloud showers will narrow it, but not below a value of $\approx 9 \mathrm{Myr}$.

We now locate the sequence of peak flux times $\tau_{j}$ for each mean period $\bar{P}$. The measure of correlation we use is

$$
\chi^{2}(\bar{P})=\frac{1}{N} \sum_{i=1}^{N}\left(\frac{T_{i}-\tau_{j}}{\bar{P} / 2}\right)_{\min }^{2} .
$$

This is constructed for each mean period using the crater agesin Table 1. These results are shown in Figure 2 for $N=5$ and 9. For crater ages which perfectly anticorrelate with the peak flux times $\chi^{2}=1$, while $\chi^{2}=0$ for perfect correlation and $\overline{\chi^{2}}=1 / 3$ for random crater ages. An alternative illustration is shown in Figure 3 in which we compare the crater ages and the peak flux times for each case.

One observes that the best correlation occurs for periods in the interval $36 \pm 2 \mathrm{Myr}$ for both the largest 5 craters and all 9 craters. A secondary signal at 30-31 Myr for the largest craters vanishes when smaller craters are included. The data are consistent with a random distribution of crater ages if the mean period is outside these intervals. These results can be compared with previous analyses in which the phase of the cycle was a free parameter and the cycle interval was uniform (Grieve and Pesonen, 1996). One can infer from Figure 3 that the best correlation for this crater data will occur within a period interval bounding $36 \mathrm{Myr}$ using any goodness of fit measure if the phase is constrained to be near the present epoch. Using different data Matsumoto and Kubotani 
0.8

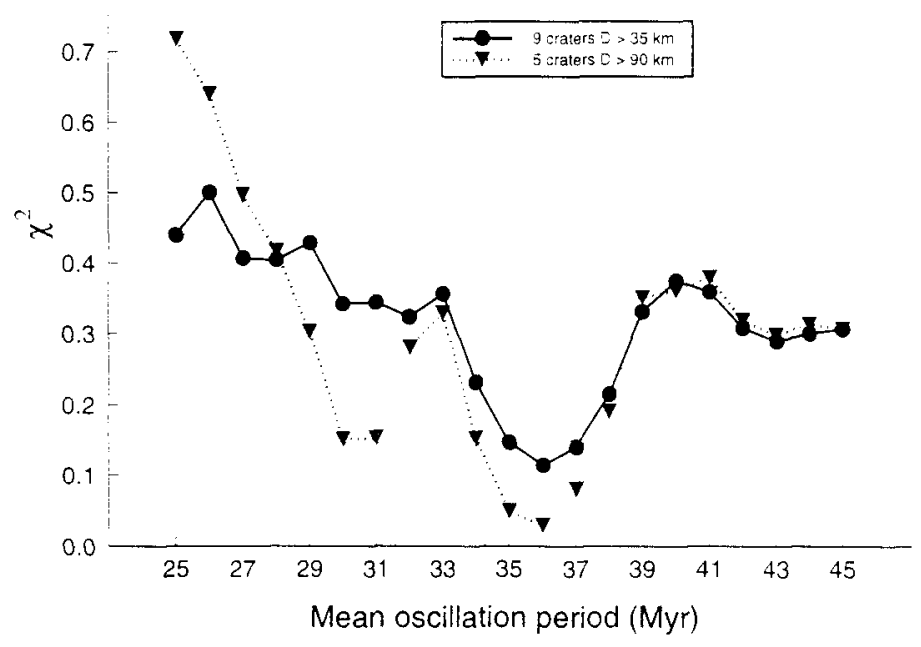

Figure 2. A correlation measure $\chi^{2}(\bar{P})$ for each of 21 mean periods in the range 25-45 Myr. Separately illustrated are the cases for the largest 5 and all 9 craters listed in Table 1.

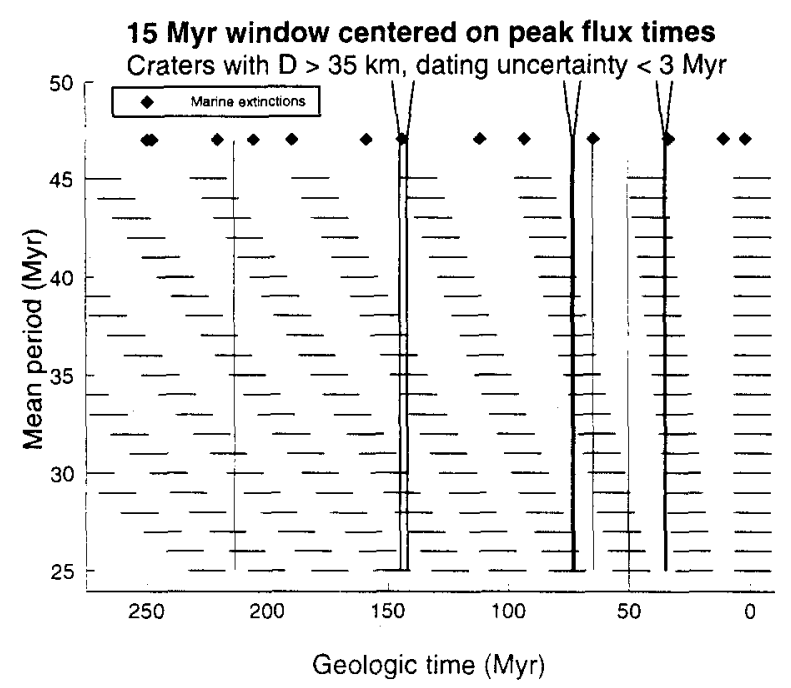

Figure 3. A comparison of 9 large accurately dated craters with a sequence of peak flux times $\tau$ for various mean periods $\overrightarrow{\vec{P}}$ as predicted by the galactic oscillations model. A $15 \mathrm{Myr}$ window is centered on each peak flux time as a reference.

(1996) have argued that there is a statistically significant correlation between peaks in cratering and extinction. This does not necessarily imply that they are quasi-periodic however.

\section{Conclusions}

Our interpretation of these results is that a galactic oscillations model in which the Solar cycle is manifest in the cratering record will be unsustainable as a working hypothesis if the cycle period is found to exclude the intervals mentioned above. Improvements in galactic disk mass determinations from stellar velocity dispersions can be obtained from Hipparcos data. Such studies are crucial in 
this regard.

What can we say if the period is found to be in these intervals? We have randomly selected a set of 9 hypothetical crater ages in a $250 \mathrm{Myr}$ interval and calculated $\chi_{\text {min }}^{2}$ for $\bar{P}=35,36$ or 37 Myr. The process was repeated 10000 times. We find that $95 \%$ of the time the random set of crater ages had $\chi_{\text {min }}^{2} \geq 0.14$. The corresponding result for 5 randomly chosen ages is $\chi^{2}{ }_{\text {min }} \geq 0.09$ for the $95 \%$ confidence level. In both cases these values exceed the correlation measure for the observed crater sets. This would not, in itself, indicate that the correlation between the oscillation cycle and the crater time series should be accepted as statistically significant. But it would indicate that such a correlation could not be readily rejected.

\section{References}

Flynn, C. and B. Fuchs 1994. Density of Matter in the Galactic Disc. MNRAS 270, 471-479.

Grieve, R.A.F. and L.J. Pesonen 1996. Terrestrial Impact Craters: Their Spatial and Temporal Distribution and Impacting Bodies. Worlds in Interaction: Small Bodies and Planets of the Solar System (H. Rickman and M.J. Valtonen, Eds.), 357-376. Kluwer, Dordrecht.

Heisler, J. 1990. Monte Carlo Simulations of the Oort Comet Cloud. Icarus 88, 104-121.

Heisler, J. and S. Tremaine 1989. How Dating Uncertainties Affect the Detection of Periodicity in Extinctions and Craters. Icarus 77, 213-219.

Matese, J.J., P.G. Whitman, K.A. Innanen and M.J. Valtonen 1995. Periodic Modulation of the Oort Cloud Comet Flux by the Adiabatically Changing Galactic Tide. Icarus 116, 255-268.

Matsumoto, M. and H. Kubotani. A Statistical Test for Correlation Between Crater Formation Rate and Mass Extinctions. MNRAS 282, 1407-1412.

Rampino, M.R., and R.B. Stothers 1986. Geological Periodicities and the Galaxy. The Galaxy and the Solar System (R. Smoluchowski, J. N. Bahcall and M. S. Matthews, Eds.), 241-260. Univ. of Arizona Press, Tucson.

Rampino, M.R., and B.M. Haggerty 1996. The "Shiva Hypothesis": Impacts, Mass Extinctions and the Galaxy. Worlds in Interaction: Small Bodies and Planets of the Solar System ( $\mathrm{H}$. Rickman and M.J. Valtonen, Eds.), 441-460. Kluwer, Dordrecht.

Rampino, M.R., Haggerty, B.M. and T.C. Pagano 1997. A Unified Theory of Impact Crises and Mass Extinctions: Quantitative Tests. Annals of the New York Academy of Sciences (J.L. Remo Ed.) 822 403-431.

Thaddeus, P. 1986. Molecular Clouds and Periodic Events in the Geologic Past. The Galaxy and the Solar System (R. Smoluchowski, J.N. Bahcall and M.S. Matthews, Eds.), 61-68. Univ. of Arizona Press, Tucson.

Shoemaker, E.M., R.F. Wolfe and C.S. Shoemaker. 1990. Asteroid and Comet Flux in the Neighborhood of the Earth. Geological Society of America Special Paper 247, 155-170. 